\title{
Involvement of urokinase in cigarette smoke extract-induced epithelial-mesenchymal transition in human small airway epithelial cells
}

\author{
Qin Wang ${ }^{1}$, Yunshan Wang ${ }^{2,3}$, Yi Zhang ${ }^{1}$, Yuke Zhang ${ }^{1}$ and Wei Xiao ${ }^{1}$
}

\begin{abstract}
Urokinase-type plasminogen activator (UPA) augments inflammation and tissue remodeling during lung injury and repair. The UPA expression in small airway epithelium of chronic obstructive pulmonary disease (COPD) increases. Epithelialmesenchymal transition (EMT) is important in the small airway fibrosis of COPD. This study shows the uPA regulation in cigarette smoke extract (CSE)-induced EMT in human small airway epithelial cell lines (HSAEpiCs). uPA is overexpressed in the small airway epithelium of COPD patients and CSE-treated cell lines. Furthermore, UPA expression correlated with vimentin expression in the small airway epithelium of COPD patients. UPA inhibition blocks CSE-induced EMT by reversing $\mathrm{E}$-cadherin and $\alpha$-catenin expression and retarding the induction of $\mathrm{N}$-cadherin and vimentin, resulting in reduction in migration. UPA overexpression in HSAEpiC cells also promotes EMT and migration. EMT is partly reversed in UPA-overexpressing HSAEpiC cells through the silencing expression of uPA receptor. In conclusion, this study provides new insights into the contribution of UPA upregulation to EMT associated with small airway remodeling in COPD.

Laboratory Investigation (2015) 95, 469-479; doi:10.1038/labinvest.2015.33; published online 23 February 2015
\end{abstract}

Epithelial-mesenchymal transition (EMT) is a process through which epithelial cells lose their epithelial features and acquire mesenchymal characteristics. EMT occurs in physiological scenarios, such as development and wound healing; EMT also demonstrates pathogenic functions, such as carcinogenesis and fibrosis in adult tissues, as a source of myofibroblasts. ${ }^{1}$ Recent studies suggest that EMT is involved in the pathogenesis of chronic obstructive pulmonary disease (COPD). ${ }^{2,3}$ Cigarette smoking induces lung remodeling in COPD. Small airway fibrosis is observed in the small airways of COPD patients because of lung remodeling, contributing to the airway obstruction of patients. ${ }^{4}$ EMT is a key step in peribronchiolar fibrosis formation.

EMT induction occurs in response to cytokines, such as the transformation of the growth factor- $\beta 1$ (TGF- $\beta 1$ ), fibroblast growth factor 2, and hepatocyte and epidermal growth factors. Despite the involvement of complex molecular mechanisms, most signals that trigger EMT downregulate E-cadherin expression through transcriptional repression or protein degradation. Recent studies have revealed that EMT can be induced by the urokinase-type plasminogen activator receptor (UPAR) and by activating uPAR-dependent cell signaling pathways. ${ }^{5}$ E-cadherin expression may also be repressed at the transcriptional level when UPAR increases snail activity. The binding of urokinase-type plasminogen activator (UPA) to UPAR promotes EMT by activating ERK1/ 2 and PI3K. An increasing body of evidence suggests that uPA may contribute to EMT. ${ }^{6}$ Transcriptional inactivation of uPA inhibits the invasion and migration of intermittent hypoxiainduced medulloblastoma cells, and vimentin expression. uPA downregulation also induces E-cadherin expression and inhibits ERK activation. ${ }^{7}$

uPA, which is more commonly known as urokinase, simply converts plasminogen into plasmin, a broad-spectrum matrix and fibrin-degrading enzyme. In addition, this protease also possesses a definite ability to stimulate cell migration and survival. These activities are exerted through the growth factor-like domain of protease that binds to UPAR and in complex transmembrane receptors, such as integrins, through the epidermal growth factor and formylpeptide receptors. ${ }^{8}$ Recent evidence suggests the possible existence of additional uPA receptor(s). ${ }^{9,10}$

Nicotinic acetylcholine receptor- $\alpha 1(\mathrm{nAChR} \alpha 1)$ is investigated as a potential fibrogenic molecule in the kidneys, given

\footnotetext{
'Department of Respiratory Medicine, Qilu Hospital, Shandong University, Jinan, China; ${ }^{2}$ Department of Anatomy, Shandong University School of Medicine, Jinan, China and ${ }^{3}$ School of Ocean, Shandong University, Weihai, China Correspondence: Professor W Xiao, PhD, Department of Respiratory Medicine, Qilu Hospital, Shandong University, 107 Wenhua Xi Road, Jinan 250012, China. E-mail: xiaowei4226@163.com 
the reports that $\mathrm{nAChR} \alpha 1$ may be an alternative classical uPAR receptor. uPA binds $n A C h R \alpha 1$ and promotes renal fibrosis. The functional knockdown of $n A C h R \alpha 1$ is shown to attenuate fibrosis significantly after ureteral obstruction, and this is a uPA-dependent effect. ${ }^{11}$ uPA deficiency reduces fibrosis in a heart damaged by viral myocarditis or left ventricular pressure overload. An increase in uPA levels is associated with numerous pathologies, such as asthma, COPD, and interstitial lung disease. ${ }^{12-14}$ The uPA mechanism of various actions remains incompletely understood despite the association of uPA with several activities.

We have recently shown that the uPA system affects the pathogenesis of COPD. The immunohistochemical staining of UPA and uPAR is significantly elevated in the small airway epithelia of COPD patients compared with that of the controls. ${ }^{15}$ Gene network search analysis shows numerous pathways that are biologically plausible candidates for COPD pathogenesis. For example, plasminogen activator, urokinase $(P L A U)$; plasminogen activator, urokinase receptor; and thrombospondin (THBS1) are three genes involved in TGF$\beta 1$ activation and matrix metalloproteinases (MMPs). ${ }^{16} \mathrm{We}$ have recently described a pathway through which cigarette smoke extract (CSE) induces EMT in human small airway epithelial cells by increasing UPAR expression and activating uPAR-dependent cell signaling pathways. ${ }^{17}$ We have investigated the effect and mechanism of uPA action on EMTs in human small airway epithelium of COPD patients, and explored the interaction between uPA and uPAR on EMTs.

\section{MATERIALS AND METHODS Patients}

Lung tissues were obtained from 75 patients (24 nonsmoker non-COPD patients, 25 smokers without COPD, and 26 smokers with COPD) at Qilu Hospital (Jinan, China) following lobectomy or pneumonectomy for various medical reasons. The diagnosis of COPD was made according to the guidelines of the Global Initiative for Chronic Obstructive Lung Disease. ${ }^{18}$ No subjects received corticosteroids (oral or inhaled) before tissue collection. All experiments were approved by the ethics committee of Qilu Hospital and informed consent was obtained from all patients before specimen collection. The clinical characteristics of the patients are shown in Table 1. All recruited patient groups were similar with regard to age. The number of pack-years was similar between smokers without COPD and smokers with COPD.

\section{Immunohistochemistry}

Serial sections $(4 \mu \mathrm{M})$ of formalin-fixed, paraffin-embedded lung tissue were used for immunohistochemical analysis. Sections were immunostained with primary antibodies recognizing uPA (ADG3689, American Diagnostica), E-cadherin (24E10, Cell Signaling Technology), and vimentin (D21H3, Cell Signaling Technology) and visualized using the avidinbiotin-peroxidase (ABC) complex (ZhongShan Biotech, Beijing, China) method. Color development was performed
Table 1 Demographic characteristic of the subjects

\begin{tabular}{lccc}
\hline & $\begin{array}{c}\text { Nonsmokers } \\
\text { (NC) } \\
n=24\end{array}$ & $\begin{array}{c}\text { Smokers } \\
\text { (NS) } \\
n=25\end{array}$ & $\begin{array}{c}\text { Smokers with } \\
\text { COPD (CS) } \\
n=26\end{array}$ \\
\hline Sex (female/male) & $17 / 7$ & $1 / 24$ & $0 / 26$ \\
Age (years) & $53 \pm 12$ & $57 \pm 11$ & $62 \pm 9$ \\
Smoking history, pack-years & - & $25 \pm 14$ & $39 \pm 29$ \\
FEV1, \% predicted & $98 \pm 15$ & $98 \pm 10$ & $71 \pm 17$ \\
FEV1/FVC \% & $85 \pm 8$ & $79 \pm 6$ & $62 \pm 5$ \\
GOLD stage & - & - & 7 \\
1 & - & - & 17 \\
2 & - & - & 2 \\
3 & - & - & - \\
4 & - & & \\
\hline
\end{tabular}

Abbreviations: COPD, chronic obstructive pulmonary disease; FEV1, forced expiratory volume in $1 \mathrm{~s}$; FVC, forced vital capacity.

Values are given as mean \pm s.d.

Pack-year $=1$ year smoking 20 cigarettes per day.

using a DAB color development kit (ZhongShan Biotech). Images were captured using an OLYMPUS DP72 light microscope (Olympus, Tokyo, Japan) fitted with a SPOT camera. Image analysis was performed using Image-Pro Plus 6.0 software (Media Cybernetics, Silver Spring, MD, USA). The area of the small airway epithelia and length of the basement membrane were evaluated. uPA and E-cadherin were expressed as mean staining density in the small airway epithelia. Vimentin was expressed as the number of positive epithelial cells $/ \mathrm{mm}$ basement membrane. All slides were analyzed in a single batch by a single experienced observer with quality assurance on randomly selected slides provided by a professional academic pathologist.

\section{Preparation of CSE}

CSE was prepared using a modified method described by Aoshiba et al. ${ }^{19}$ Briefly, one filterless commercial cigarette (13 mg of tar and $1.2 \mathrm{mg}$ of nicotine per cigarette) was combusted using a modified, syringe-driven apparatus. Mainstream smoke was bubbled through $20 \mathrm{ml}$ of serum-free Ham's F12 nutrient medium (F12). The resulting suspension was adjusted to $\mathrm{pH} 7.4$ with concentrated $\mathrm{NaOH}$ and filtered through a $0.22-\mu \mathrm{m}$ pore filter to remove bacteria and large particles. This solution (designated as a 100\% CSE solution) was used within $30 \mathrm{~min}$ of preparation.

\section{Cell Culture}

Human small airway epithelial cells (HSAEpiCs) were obtained from ScienCell Research Laboratories (Cat. No. 3231). Cells were cultured in small airway epithelial cell medium (SAEpiCM) at $37^{\circ} \mathrm{C}$ in a water-saturated atmosphere with $5 \%$ carbon dioxide. 


\section{Vector Construction and Cell Transfection}

HSAEpiC cells that overexpress human uPA were prepared by transfecting cells with pWZL-uPA or with empty vector using Lipofectamine 2000 (Invitrogen, Carlsbad, CA, USA) in accordance with the manufacturer's instructions. Cells were trypsinized and subjected to various experiments. The expression of uPA was confirmed by western blotting.

\section{UPAR-Specific Short Hairpin RNA Inhibition}

For knockdown of uPAR expression in HSAEpiCs, short hairpin RNAs (shRNAs) targeting human uPAR (5'-GGUG AAGAAGGGCGUCCAA-3') were cloned into the pSuper vector as previously described. ${ }^{5}$ Control oligonucleotides corresponding to the inverse UPAR shRNA sequences were also prepared. Cells were transfected with pSuper-shRNA targeting uPAR or empty vector using Lipofectamine2000. uPAR knockdown was assessed by western blotting.

\section{Cell Migration Assay}

Cell migration assay was done using Transwell (6.5-mm diameter, $8-\mu \mathrm{m}$ pore size polycarbonate membrane) that was obtained from Corning (Cambridge, MA, USA). Cells were treated without or with $5 \%$ CSE, and 5\% CSE $+20 \mathrm{nmol} / \mathrm{l}$ amiloride before adding the cells to the top chamber $\left(1 \times 10^{5} /\right.$ well $)$. Cells were transfected with constructs encoding empty vector or uPA in the upper chamber of Transwells. F12 medium with $10 \%$ fetal bovine serum was used in the lower chamber. After $24 \mathrm{~h}$, the nonmigrating cells were removed from the upper surface of each Transwell by a cotton swab. Transwell membranes were then stained with $0.5 \%$ crystal violet. Cells that migrated through the membrane to the lower surface were counted.

\section{Real-Time Reverse Transcription-PCR}

Total RNA was extracted using Trizol reagent (Invitrogen) according to the manufacturer's instructions. First-strand cDNA was synthesized using SuperScript II reverse transcriptase (Invitrogen). Real-time PCR reactions were prepared using SYBR ${ }^{\circledR}$ Green Real-Time PCR Master Mix (Invitrogen) and PCR was performed with an ABI PRISM7900 HT Sequence Detection System. PCR was performed using the following primers: PLAU forward 5'-GCGACTCCAAAGGC AGCAATGAACT-3' and reverse $5^{\prime}$-TCTGCGCAGTCATGCA CCATGC- $3^{\prime}$, GAPDH forward $5^{\prime}$-GCTGGCGCTGAGTACGT CGT-3' and reverse ${ }^{\prime}{ }^{\prime}$-ACGTTGGCAGTGGGGACACG- $3^{\prime}$. Endogenous GAPDH was used as a normalization control. Results were analyzed by the relative quantity $(\Delta \Delta \mathrm{Ct})$ method. Experiments were performed in triplicate with internal duplicate determinations.

\section{Western Blot Analysis}

Cells extracts were prepared using ice-cold RIPA buffer containing complete protease inhibitor cocktail (Roche) and $1 \mathrm{mM}$ sodium orthovanadate. Proteins were separated by $10 \%$ SDS-PAGE and transferred to PVDF membranes and probed with antibodies detecting human uPA, uPAR (10G7, Santa Cruz), E-cadherin, vimentin, $\alpha$-catenin (610194, BD Transduction Laboratories), and N-cadherin (610920, BD Transduction Laboratories). $\beta$-Actin (4970, Cell Signaling Technology) was used as an endogenous loading control.

\section{Statistical Analysis}

The statistical software packages (Graph Pad Prism and SPSS 13.0) were used for the analysis. The Mann-Whitney $U$-test was used for comparisons between patient groups. Spearman's test was used for correlation analyses. When a significant difference was found, Bonferroni-corrected Mann-Whitney $U$-tests were used. Student's $t$-test was used for in vitro experiments on HSAEpiCs. All results are presented as mean \pm s.e.m. The $P$-values of $<0.05$ were accepted as statistically significant.

\section{RESULTS \\ UPA and EMT Biomarker Expression in the Small Airway Epithelium of COPD Subjects}

To explore whether uPA expression is increased in small airway epithelial cells, lung tissue samples were obtained from smokers with COPD, smokers without COPD, and nonsmoker non-COPD subjects. The uPA expression in small airway epithelium was evaluated by immunohistochemical analysis of lung sections. Figure 1a presents a representative staining pattern that shows a more intense uPA staining in the epithelium of the small airways in subjects with COPD and smokers without COPD than nonsmoker non-COPD controls, especially in COPD subjects. The quantitative analysis of uPA staining showed that the uPA expression was significantly increased in COPD when compared with nonsmoker $(P<0.01)$ and smoker controls $(P<0.01)$. The uPA expression was higher in smoker controls than in nonsmokers $(P<0.05)$.

We also observed the expression of E-cadherin (Figure 2d$\mathrm{f}$ and $\mathrm{j}$ ) and vimentin (Figure $2 \mathrm{~g}-\mathrm{i}$ and $\mathrm{k}$ ) in lung sections of COPD subjects and control subjects. We observed a significant immunostaining of E-cadherin in small airway epithelial cells from a nonsmoker and a smoker, whereas E-cadherin expression was partly decreased in a COPD subject. Small airway epithelial cells from the same COPD subject and from the same smoker had significant expression of vimentin and $\mathrm{uPA}$. Vimentin-positive cells were increased in the epithelium of small airways in COPD patients when compared with controls. In contrast, E-cadherin staining was significantly reduced in the small airway epithelia of patients with COPD compared with controls.

\section{Correlations}

We have assessed whether the uPA expression in small airways is correlated with $\mathrm{FEV}_{1} \%$ or vimentin expression in the small airways. No correlation was observed between the uPA expression in the small airways and $\mathrm{FEV}_{1} \%$ (Figure $3 \mathrm{a}-\mathrm{c}$ ). The correlation analyses show that uPA expression in small airways was correlated with vimentin expression in the 

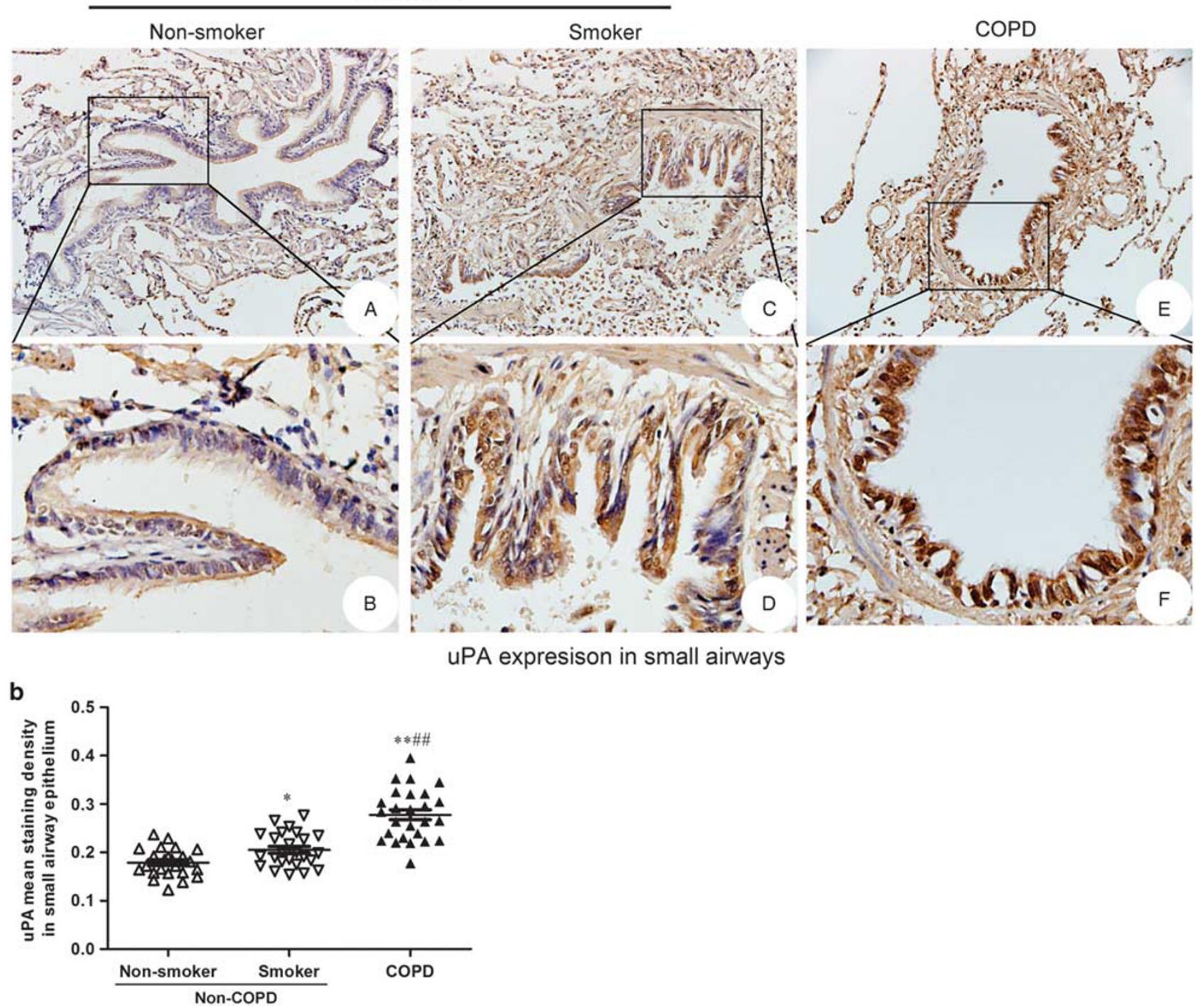

Figure 1 uPA immunostaining in small airways epithelium with chronic obstructive pulmonary disease (COPD). Immunohistochemistry assessment of small airway epithelium for expression of uPA in nonsmoker subjects $(n=24)$, smokers $(n=25)$, and COPD subjects $(n=26)$. (a) Representative uPA immunostaining (brown stain) in small airways of a nonsmoker (A, B), a smoker (C, D), and a COPD patient (E, F). (b) Quantification of uPA protein levels in small airways by mean staining density in small airways. ${ }^{*} P<0.05,{ }^{* *} P<0.01$ compared with nonsmokers. ${ }^{\# \#} P<0.01$ compared with smokers.

COPD $(r=0.5657, P<0.001)$ and smoker groups $(r=0.2581$, $P<0.05)$ but not in the nonsmoker group (Figure $3 \mathrm{~d}-\mathrm{f}$ ).

Increased uPA Expression in CSE-Induced HSAEpiC Cells We previously demonstrated that activation of uPARdependent cell signaling promoted EMT of HSAEpiCs by CSE. The uPA interacts with its receptor, uPAR, to regulate migration, chemotaxis, and cytokine production. ${ }^{20}$ Therefore, we tested whether uPA was upregulated in CSEinduced HSAEpiCs. To address this question, we incubated these cells with different concentrations of CSE for $72 \mathrm{~h}$ and analyzed uPA expression at both mRNA and protein levels. HSAEpiC cells had dose-dependent mRNA and protein expression of uPA in response to stimulation with different concentrations of CSE (Figure $4 \mathrm{a}$ and $\mathrm{b}$ ). HSAEpiC cells were later incubated with $5 \%$ CSE for 0 to $72 \mathrm{~h}$ and then uPA mRNA and protein was measured by real-time PCR and western blotting. Figure $4 \mathrm{c}$ and $\mathrm{d}$ showed that CSE induced uPA expression in a time-dependent manner. The effect becomes apparent at $48 \mathrm{~h}$. In our previous study, we show that UPAR expression was increased following CSE exposure in HSAEpiC intracellular. As uPAR has multiple isoforms including a soluble form, we measured using ELISA the soluble uPA receptor (suPAR) in supernatants of different cell cultures with or without CSE treatment. The results showed that CSE could increase the level of suPAR in the super- 

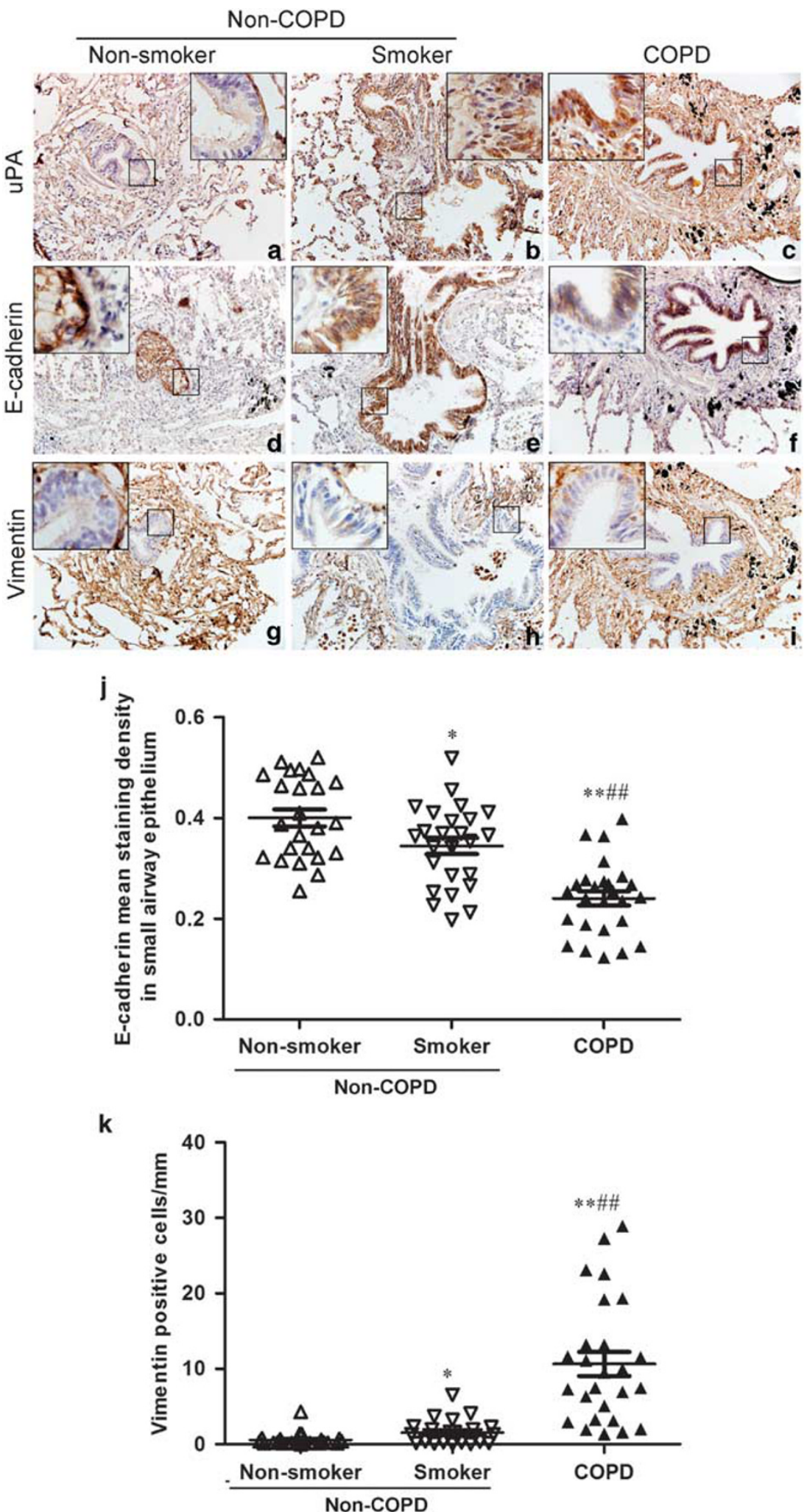

Figure 2 uPA and EMT biomarkers in small airways epithelium of patients with COPD. Representative uPA (a, b, c), E-cadherin (d, e, f), and vimentin $(\mathbf{g}, \mathbf{h}, \mathbf{i})$ immunostaining (brown stain) in small airways of a nonsmoker, a smoker, and a COPD patient with serial lung sections. (j) Quantification of E-cadherin protein levels in the small airways epithelium by mean staining density in small airways. (k) Quantification of vimentin protein levels in the small airways epithelium by the number of positive epithelial cells per mm reticular basement membrane (Rbm) in small airways. ${ }^{*} P<0.05$, ${ }^{* *} P<0.01$ compared with nonsmokers: ${ }^{\# \#}<0.01$ compared with smokers. 
a
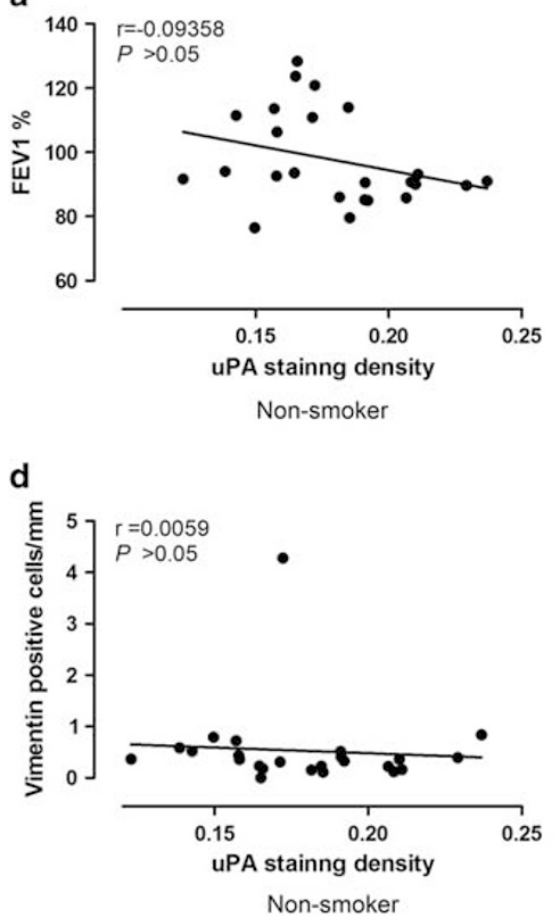

b

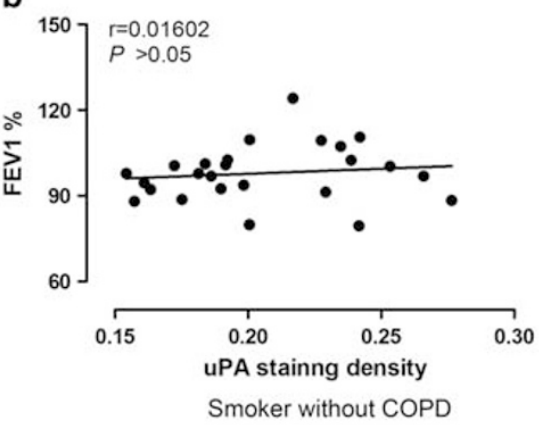

e

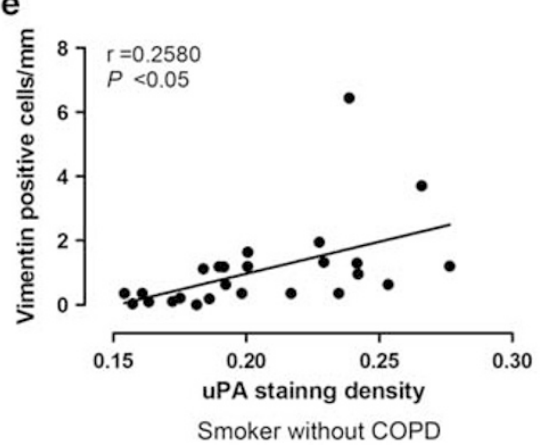

C

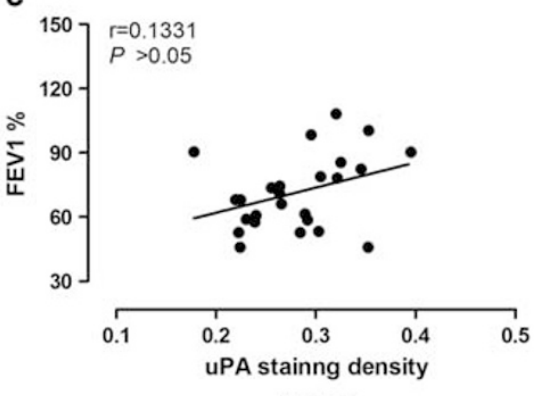

COPD

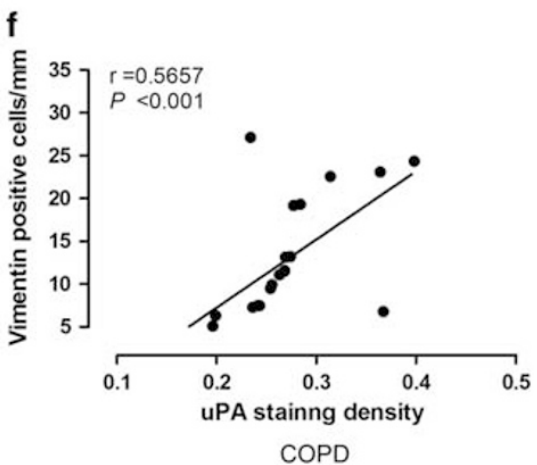

Figure 3 Correlations between the expression of uPA and lung functional parameters or vimentin-positive cells in small airways of nonsmokers $(n=24)$, smokers $(n=25)$, and COPD subjects $(n=26)$. The expression of uPA in small airways of nonsmokers $(\mathbf{a})$, smokers $(\mathbf{b})$, and COPD subjects (c) was not correlated with $\mathrm{FEV}_{1} \%$ of predicted. The expression of uPA in small airways of nonsmokers (d) was not correlated with vimentin-positive cells per $\mathrm{mm}$ in small airways. The expression of uPA in small airways of smokers (e) and COPD subjects (f) was related with vimentin-positive cells per $\mathrm{mm}$ in small airways by Spearman's correlation test.

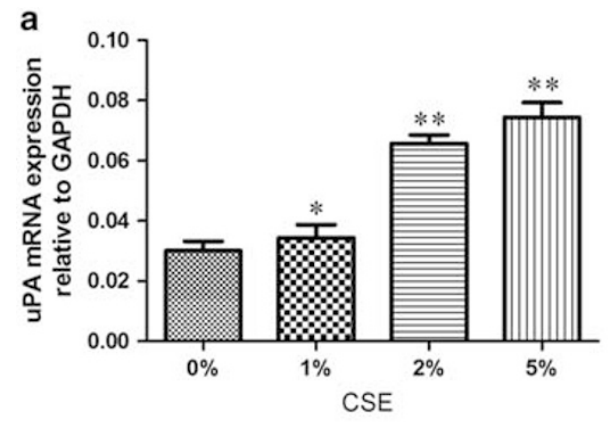

b

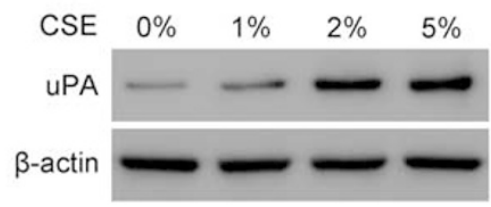

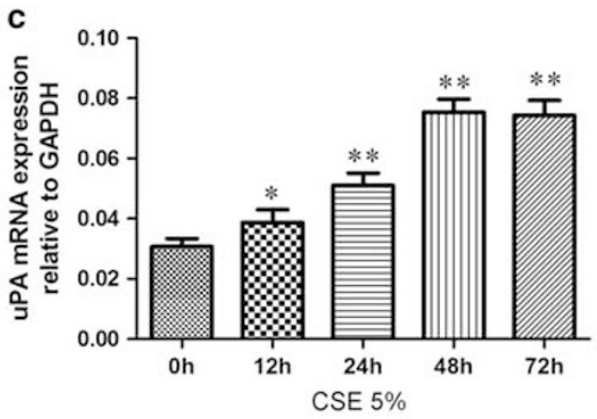

d

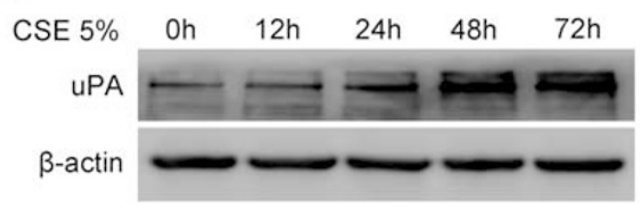

Figure 4 Cigarette smoke extract (CSE) increases uPA expression in cultured human small airway epithelial cells (HSAEpiC). Cells were treated with either medium alone or CSE. The expression levels of UPA mRNA (a) and protein (b) were examined by real-time PCR and western blotting with variable concentrations of CSE after $72 \mathrm{~h}$ in HSAEpiC cells. The expression levels of uPA mRNA (c) and protein (d) were examined by real-time PCR and western blotting with 5\% CSE for indicated times in HSAEpiC cells. ${ }^{*} P<0.05$ compared with control, ${ }^{* *} P<0.01$ compared with control. All results are from three independent experiments. Error bar indicate s.d. 
a

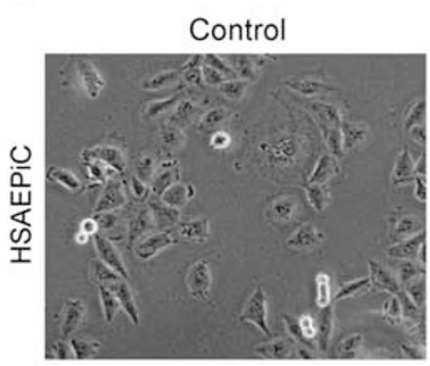

C

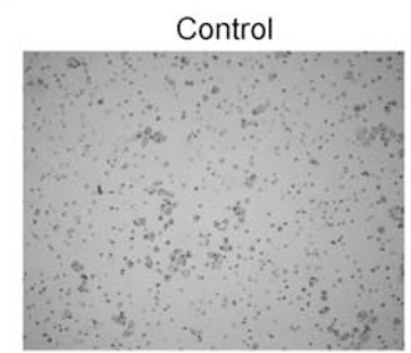

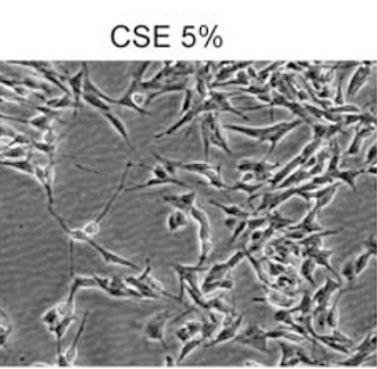
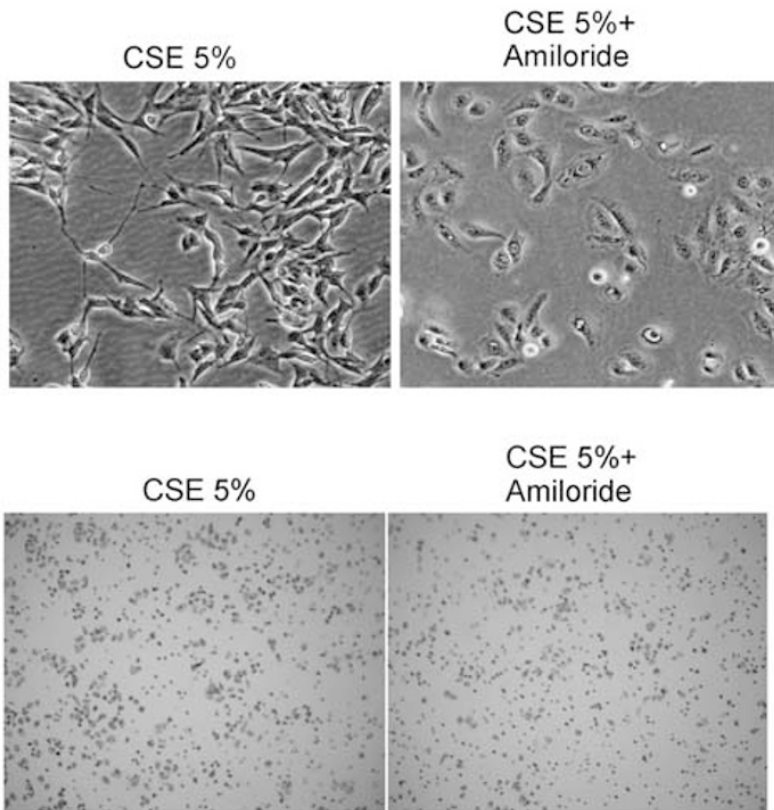
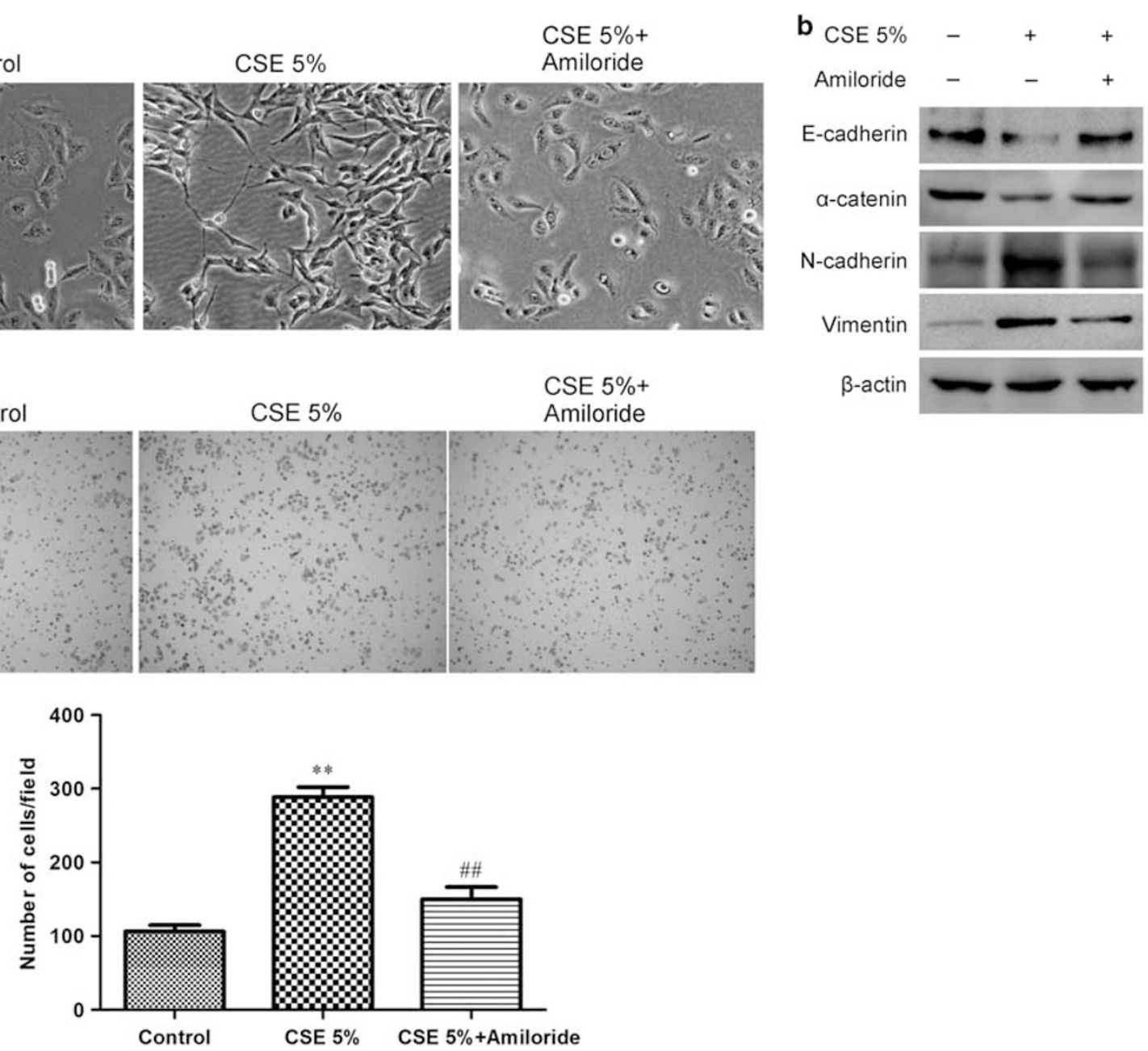

Figure 5 uPA is necessary for CSE-induced EMT. $(\mathbf{a}, \mathbf{b})$ HSAEpiC cells treated with CSE were cultured for $72 \mathrm{~h}$ with or without uPA inhibitor amiloride $(20 \mathrm{nmol} / \mathrm{l})$. (a) Cell images were captured using phase-contrast microscopy. (b) Cell extracts were subjected to western blotting to detect E-cadherin, $\alpha$-catenin, $\mathrm{N}$-cadherin, and vimentin. (c) Cells were allowed to migrate in Transwell chamber for $24 \mathrm{~h}$ with or without amiloride. ${ }^{*} P<0.01$ compared with control. ${ }^{\# \#} P<0.01$ compared with cells with 5\% CSE. All results are from three independent experiments. Error bar indicate s.d.

natants in both concentration- and time-dependent manner (Supplementary Figure 1). These results are consistent with our previous reports in intracellular that UPAR is increasing in CSE-induced cells. ${ }^{17}$

\section{UPA Is Required for CSE-Induced EMT in HSAEpiC Cells}

To test whether uPA is responsible for CSE-induced EMT, we treated HSAEpiC cells with $20 \mathrm{nmol} / \mathrm{l}$ amiloride (for uPA inhibition). Figure $5 \mathrm{a}$ shows that after $72 \mathrm{~h}$ with $5 \%$ CSE, HSAEpiC cells, which typically appear epithelial with welldeveloped cell junctions, acquired a spindle shape and generally lost cell contacts. Amiloride preserved epithelial cell morphology and cell contacts in HSAEpiC cells that were exposed to CSE, as determined by phase-contrast imaging.

As expected, the CSE-mediated EMT was observed in HSAEpiC cells treated with CSE. We exposed HSAEpiC cells to $5 \%$ CSE for $72 \mathrm{~h}$. These cells demonstrated increased
$\mathrm{N}$-cadherin and vimentin expression and loss of E-cadherin and $\alpha$-catenin. HSAEpiC cells underwent EMT, as determined by western blotting. Figure $5 \mathrm{~b}$ shows that in the presence of both amiloride and CSE, cells had increased levels of E-cadherin and $\alpha$-catenin and decreased levels of $\mathrm{N}$-cadherin and vimentin expression as compared with control cells treated with CSE.

Exposure of HSAEpiC cells to CSE promotes cell migration. To test whether CSE-induced cell migration is reversed by amiloride, we examined it by Transwell assays. Figure $5 c$ shows that the amiloride inhibited the increase in cell migration observed in HSAEpiC cells treated with CSE alone $(P<0.01)$.

\section{UPA Overexpression Induces EMT in HSAEpiC Cells}

Although the data presented thus far indicated that uPA was necessary for EMT in CSE-induced HSAEpiC cells, we wished 
a

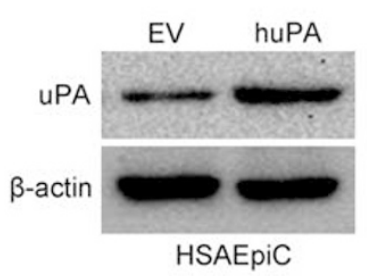

b

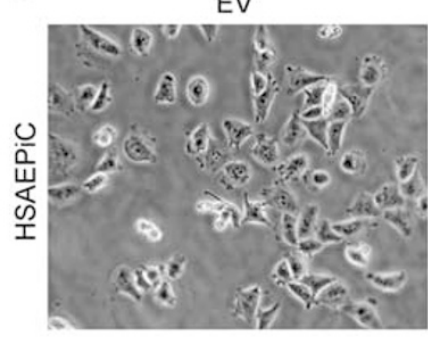

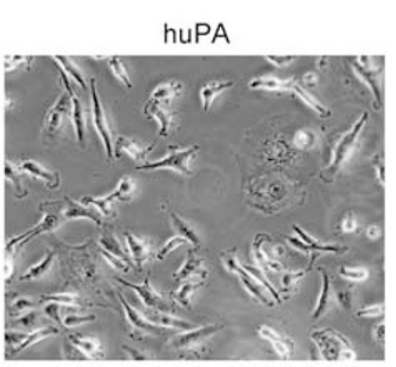

c

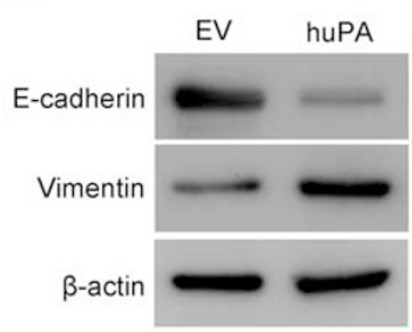

d
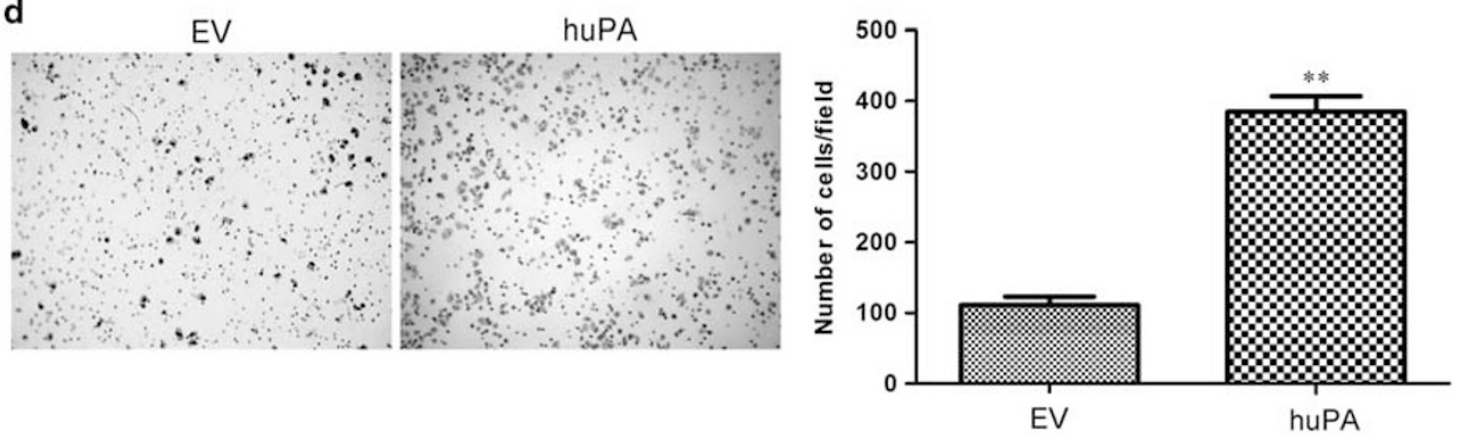

Figure 6 uPA overexpression induces EMT in HSAEpiC cells. HSAEpiC cells were transfected to transiently express uPA or empty vector. (a) Cells extracts were subjected to western blotting to detect uPA. (b) Cell images were captured using phase-contrast microscopy. (c) Cell extracts were subjected to western blotting to detect E-cadherin and vimentin. (d) Cells were allowed to migrate in Transwell chamber for $24 \mathrm{~h}$. ${ }^{* *} P<0.01$ compared with control. All results are from three independent experiments. Error bar indicate s.d.

to test whether uPA alone induces EMT in HSAEpiC cells. To accomplish this goal, we overexpressed UPA in HSAEpiC cells and studied these cells without CSE. Initially, we performed transient transfection experiments, introducing a full-length human uPA expression construct in pWZL. Control cells were transfected with empty vector alone. Figure 6a shows that the level of uPA protein was substantially increased in uPA-overexpressing HSAEpiC cells as compared with control cells transfected with empty vector (EV).

We hypothesized that uPA may be necessary for morphologic and molecular parameters of EMT. To test this hypothesis, we used uPA-overexpressing and control HSAEpiC cells. Phase-contrast imaging of uPA-overexpressing HSAEpiC cells revealed loss of cell contacts and they acquired a spindle shape (Figure 6b). uPA-overexpressing cells induced expression of vimentin and decreased E-cadherin expression, as determined by western blotting (Figure 6c). Figure 6d shows that transient transfection with uPA increased cell migration compared with control cells $(P<0.01)$. These results indicated that uPA overexpression independently induced EMT in HSAEpiC cells, without exposure to CSE.

\section{UPAR Gene Silencing Reverses EMT in uPA-Overexpressing HSAEpiC Cells}

uPA binding to uPAR is necessary for leading to an extracellular protease cascade implicated in mechanisms including cell migration, MMP activation, and intracellular signaling molecules. ${ }^{21,22}$ We hypothesized that endogenous production of uPAR in uPA-overexpressing HSAEpiC cells may be necessary to sustain EMT. To test this hypothesis, we transfected uPA-overexpressing and control HSAEpiC cells with the uPAR shRNA silencing vector, pSuper-shuPAR. Control cells were transfected with empty vector (pSuper). uPAR protein expression was measured $48 \mathrm{~h}$ later. As shown in Figure $7 \mathrm{a}-\mathrm{c}$, uPA-overexpressing cells that were transfected without or with pSuper demonstrated increased levels of uPAR protein compared with control empty vector cells. pSuper-shuPAR decreased uPAR protein expression compared with control uPA-overexpressing cells $(P<0.01)$. pSuper-shuPAR also partially decreased vimentin protein expression and increased E-cadherin protein expression compared with control uPA-overexpressing cells $(P<0.01)$, suggesting that UPAR gene silencing may partly reverse uPAmediated EMT (Figure 7d-f).

\section{DISCUSSION}

We hypothesized that a predisposition to altered uPA expression may be associated with EMT in COPD. Through a series of controls and individuals with COPD, we showed that $\mathrm{UPA}$ expression is increased in the bronchioles of subjects with COPD, particularly in the epithelium. The relationship between uPA expression in the epithelium and small airway remodeling requires further investigation, but relating epithelial histology to uPA expression in a crosssectional study would be difficult to interpret. Our previous studies found that uPA staining increased in the small airway 

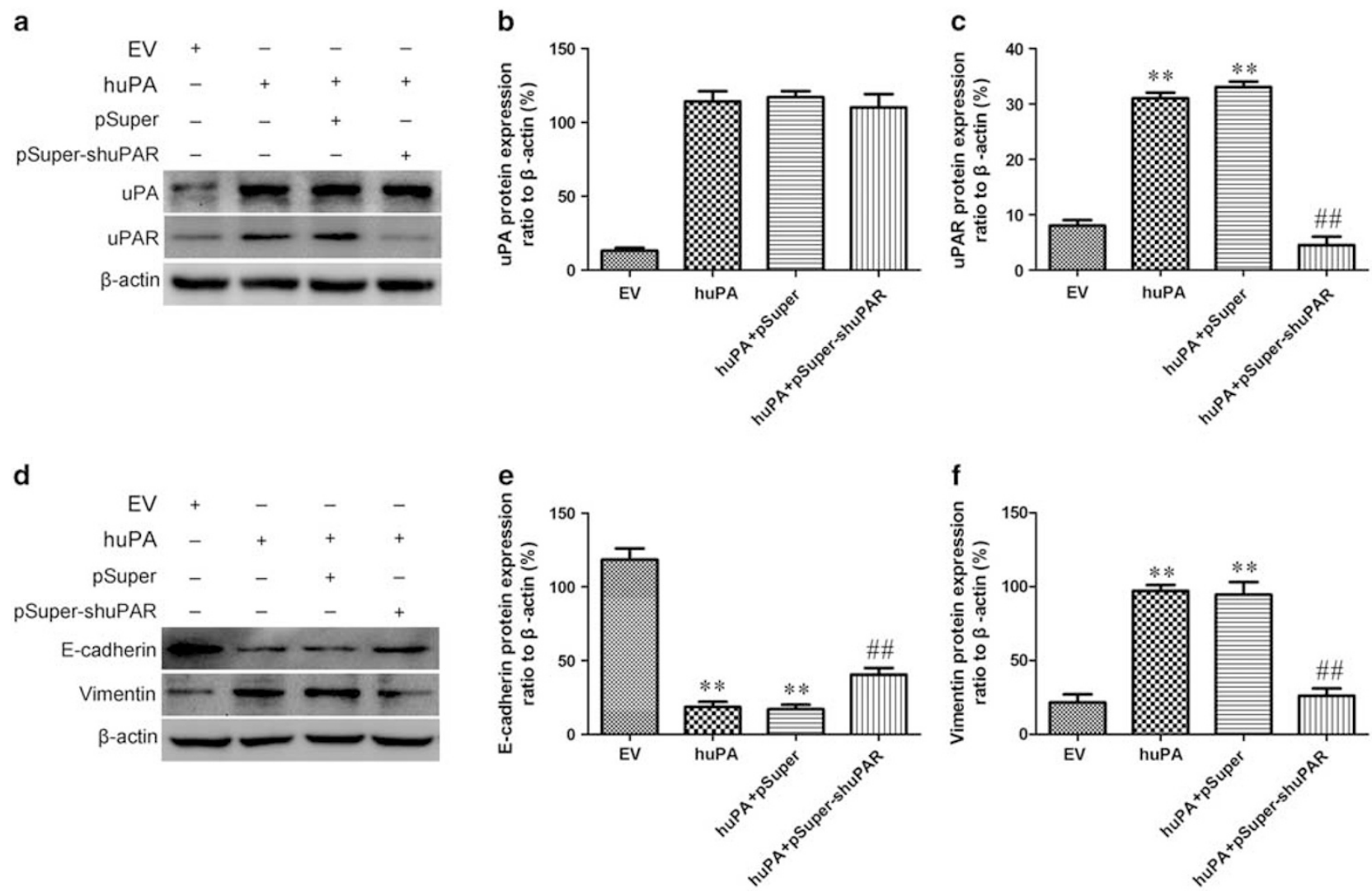

Figure 7 uPAR gene silencing reverses EMT in uPA-overexpressing HSAEpiC cells. Control (EV) and uPA-overexpressing (huPA) HSAEpiC cells were transfected with pSuper or pSuper-shuPAR. (a-c) Cells extracts were subjected to western blotting to detect uPA and uPAR. (d-f) Cells extracts were subjected to western blotting to detect E-cadherin and vimentin. ${ }^{*} P<0.01$ compared with EV. ${ }^{\# \#} P<0.01$ compared with cells with huPA + pSuper. All results are from three independent experiments. Error bar indicate s.d.

epithelium of 16 COPD patients who underwent surgical resection compared with controls. ${ }^{15}$ This study is the first to investigate the uPA protein expression ex vivo in the small airways of a number of control and COPD individuals. These findings provide a basis for further investigation of the role of uPA in the epithelium. Using cultured human HSAEpiC cells, we have shown that alteration in the uPA pathway is one cause of small airway fibrosis in COPD pathology.

COPD is a complex condition of both airway and lung parenchymal components, and is caused by the persistent inhalation of toxic particles and gases that are primarily but not exclusively derived from tobacco smoke. ${ }^{23}$ It is of interest that different regions respond in different ways; for example, the small conducting airways $<2 \mathrm{~mm}$ in diameter become the major site of airway obstruction in COPD. ${ }^{24}$ In contrast, dilatation and destruction of the gas-exchanging tissues beyond the terminal bronchioles define emphysematous destruction of the lung. The new data based on micro-CT imaging indicate that both narrowing and reduction in the number of terminal bronchioles contribute to the rapid decline in $\mathrm{FEV}_{1}$, leading to severe airway obstruction in COPD. ${ }^{25}$ Moreover, the observation that terminal bronchiolar changes before the onset of emphysematous destruction suggests that this destruction begins in the early stages of COPD. EMT is associated with fibrosis that occurs in the lung tissues. ${ }^{26}$ EMTrelated changes are described in other chronic epithelial stress, and are also present in the airway epithelia of COPD.,27

Several signaling factors have emerged as mediators of EMT that is associated with small airway fibrosis in COPD. Previous data support the hypothesis that TGF- $\beta 1$ induced by CSE activates Smad3 and ERK1/2, contributing to the EMT process in differentiated human bronchial epithelial cells. ${ }^{27}$ We have recently shown that increased uPAR expression in the small airway epithelium of COPD patients participates in an active EMT process modulated by PI3K/ Akt and GSK3 $\beta .{ }^{17}$ uPAR is a glycosyl phosphatidylinositolanchored protein with a high affinity to uPA. The uPAR expression and its ability to bind uPA are required for signaling. ${ }^{28}$ To the best of our knowledge, the possibility of achieving this EMT process by uPA has not been previously addressed. Therefore, we aim to determine whether uPA contributed to EMT in small airway epithelium in COPD.

Some cancer cells demonstrate an increased uPA in EMT process, but do not elaborate its effects to EMT. ${ }^{29}$ In our study, the increasing expression of uPA was caused by exposure to CSE. In addition, amiloride, a UPA inhibitor, signifi- 
cantly reduced migration of HSAEpiC cells, and caused a greater inhibition of CSE-mediated EMT. The uPA-overexpressing HSAEpiC cells acquired a fibroblast-like morphology and generally lost cell contacts accompanied by EMT. These data showed that uPA regulated the process of EMT in the small airway epithelial cell line. The ability of uPA to markedly induce EMT in HSAEpiC cells in vitro and the increased expression of uPA in small airways of COPD subjects demonstrate an intricate link between airway remodeling and uPA pathway and potentially substantial pathophysiological significance.

In lung (patho) physiology, uPA augments inflammation and tissue remodeling. ${ }^{30}$ Targeting uPA to treat inflammation and tissue remodeling in respiratory disease is less developed, and there is scant preclinical evidence regarding the likely effectiveness of this approach. Shetty et al ${ }^{31}$ showed that uPAmediated induction of TF occurs in lung epithelial cells to promote increased fibrin deposition in the airways during acute lung injury. Enhancing uPA/plasmin activity lessens the airway remodeling in a murine asthma model. ${ }^{32}$ This remains the case, even though there is compelling evidence of uPA involvement in tissue remodeling, and uPA has attracted attention as an emerging therapeutic target in cancer. ${ }^{33}$ Genetic studies have also shown an essential role of uPA in tissue healing or regeneration. For example, the loss of uPA impaired scar formation, because wound fibroblasts were unable to activate the fibrogenic TGF- $\beta 1$. Using phage display technology, Liang et $a l^{34}$ reported putative uPA-binding consensus sequences in 12 transmembrane receptors and suggested them as candidate alternative uPA receptors. Among these candidate receptors, several are already known as uPAR co-receptors: low-density lipoprotein receptor-related protein, gp130, integrin $\alpha \mathrm{v}$, uPAR-associated protein (also known as Endo180 and Mrc2), and the insulin-like growth factor II/mannose 6-phosphate receptor. It is also possible that different uPA domains might simultaneously bind to uPAR and one of its co-receptors. ${ }^{35}$

Based on this information, a role for uPAR with uPA signaling, however, cannot be excluded. The elevated levels of UPAR in small airway epithelia and alveolar wall cells were also observed in COPD patients with a significant emphysema in our previous study. ${ }^{15}$ Lungs from $\operatorname{uPAR}(-/-)$ mice displayed nonspecific interstitial pneumonia-like pathological features, with a significant increase in alveolar septal width and collagen content. ${ }^{36}$ These studies suggest that downregulation of UPAR may be related with interstitial pulmonary fibrosis; however, the uPAR-initiated EMT in small airway of COPD patients may be accounting for small airway fibrosis in COPD. It is of interest that this phenomenon may be associated with pathological characteristics of different regions in COPD. In the present study we investigated the effect of uPA on EMT by downregulation of uPAR expression. The effect of uPA involved in the EMT of the cultured HSAEpiCs was partly dependent on the presence of uPAR. The results presented in this study suggest that in cell culture, induction of EMT by uPA requires that the small airway epithelial cells not only express high levels of uPA but also uPAR, so that UPAR is ligated and uPARdependent cell signaling is activated. If this pathway is operational, then activation of uPA/uPAR-dependent EMT may represent a novel mechanism by which the small airway epithelial cell microenvironment affects airway remodeling progression.

In summary, in this study we have made the observation that small airway epithelial cells express uPA by immunohistology. The potential disease relevance of uPA expression in the small airway epithelium is suggested from our studies demonstrating that uPA is highly expressed in the small airways of COPD subjects and correlates with the expression of vimentin in small airways of smokers and COPD groups. Further studies in vitro showed high levels of uPA induced by CSE in HSAEpiCs that may be responsible for CSE-induced EMT. Upon silencing of uPAR gene in uPA-overexpressing HSAEpiC cells, the uPA-induced EMT is reduced. Overall, these studies suggest that uPA expression by small airway epithelium may modulate the small airway remodeling in COPD through EMT.

Supplementary Information accompanies the paper on the Laboratory Investigation website (http://www.laboratoryinvestigation.org)

\section{ACKNOWLEDGMENTS}

This work was supported by the National Natural Science Foundation of PR China (No. 81370148) and Shandong Provincial Natural Science Foundation of PR China (Y2007C104).

\section{DISCLOSURE/CONFLICT OF INTEREST}

The authors declare no conflict of interest.

1. Kalluri R, Weinberg RA. The basics of epithelial-mesenchymal transition. J Clin Invest 2009;119:1420-1428.

2. Kalita M, Tian B, Gao B, et al. Systems approaches to modeling chronic mucosal inflammation. Biomed Res Int 2013;2013:505864.

3. Sohal SS, Reid D, Soltani A, et al. Reticular basement membrane fragmentation and potential epithelial mesenchymal transition is exaggerated in the airways of smokers with chronic obstructive pulmonary disease. Respirology 2010;15:930-938.

4. Sohal SS, Ward C, Danial W, et al. Recent advances in understanding inflammation and remodeling in the airways in chronic obstructive pulmonary disease. Expert Rev Respir Med 2013;7:275-288.

5. Lester RD, Jo M, Montel V, et al. uPAR induces epithelial-mesenchymal transition in hypoxic breast cancer cells. J Cell Biol 2007;178:425-436.

6. Jo M, Lester RD, Montel V, et al. Reversibility of epithelialmesenchymal transition (EMT) induced in breast cancer cells by activation of urokinase receptor-dependent cell signaling. J Biol Chem 2009;284:22825-22833.

7. Gupta R, Chetty C, Bhoopathi P, et al. Downregulation of UPA/uPAR inhibits intermittent hypoxia-induced epithelial-mesenchymal transition (EMT) in DAOY and D283 medulloblastoma cells. Int J Oncol 2011;38:733-744.

8. Schuliga M, Westall G, Xia Y, et al. The plasminogen activation system: new targets in lung inflammation and remodeling. Curr Opin Pharmacol 2013;13:386-393.

9. Koopman JL, Slomp J, de Bart AC, et al. Mitogenic effects of urokinase on melanoma cells are independent of high affinity binding to the urokinase receptor. J Biol Chem 1998;273:33267-33272. 
10. Mukhina S, Stepanova V, Traktouev D, et al. The chemotactic action of urokinase on smooth muscle cells is dependent on its kringle domain. Characterization of interactions and contribution to chemotaxis. J Biol Chem 2000;275:16450-16458.

11. Zhang G, Kernan KA, Thomas A, et al. A novel signaling pathway: fibroblast nicotinic receptor alpha1 binds urokinase and promotes renal fibrosis. J Biol Chem 2009;284:29050-29064.

12. Bhandary YP, Shetty SK, Marudamuthu AS, et al. Regulation of lung injury and fibrosis by p53-mediated changes in urokinase and plasminogen activator inhibitor-1. Am J Pathol 2013;183:131-143.

13. Stewart CE, Sayers I. Urokinase receptor orchestrates the plasminogen system in airway epithelial cell function. Lung 2013;191:215-225.

14. Xiao W, Hsu YP, Ishizaka A, et al. Sputum cathelicidin, urokinase plasminogen activation system components, and cytokines discriminate cystic fibrosis, COPD, and asthma inflammation. Chest 2005;128:2316-2326.

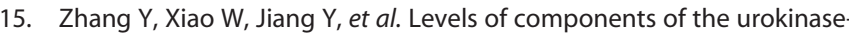
type plasminogen activator system are related to chronic obstructive pulmonary disease parenchymal destruction and airway remodelling. J Int Med Res 2012;40:976-985.

16. Wang IM, Stepaniants S, Boie Y, et al. Gene expression profiling in patients with chronic obstructive pulmonary disease and lung cancer. Am J Respir Crit Care Med 2008;177:402-411.

17. Wang $Q$, Wang $Y$, Zhang $Y$, et al. The role of UPAR in epithelialmesenchymal transition in small airway epithelium of patients with chronic obstructive pulmonary disease. Respir Res 2013;14:67.

18. Vestbo J, Hurd SS, Agusti AG, et al. Global strategy for the diagnosis, management, and prevention of chronic obstructive pulmonary disease: GOLD executive summary. Am J Respir Crit Care Med 2013;187:347-365.

19. Aoshiba K, Tamaoki J, Nagai A. Acute cigarette smoke exposure induces apoptosis of alveolar macrophages. Am J Physiol Lung Cell Mol Physiol 2001;281:L1392-L1401.

20. Schmitt M, Wilhelm O, Janicke $F$, et al. Urokinase-type plasminogen activator (UPA) and its receptor (CD87): a new target in tumor invasion and metastasis. J Obstet Gynaecol (Tokyo 1995) 1995;21:151-165.

21. Blasi F, Carmeliet P. uPAR: a versatile signalling orchestrator. Nat Rev Mol Cell Biol 2002:3:932-943.

22. Stewart $\mathrm{CE}$, Nijmeh HS, Brightling $\mathrm{CE}$, et al. UPAR regulates bronchial epithelial repair in vitro and is elevated in asthmatic epithelium. Thorax 2012;67:477-487.

23. Vestbo J, Hurd SS, Agusti AG, et al. Global strategy for the diagnosis, management and prevention of chronic obstructive pulmonary disease, GOLD Executive Summary. Am J Respir Crit Care Med 2013;187:347-365

24. Hogg JC, Chu F, Utokaparch S, et al. The nature of small-airway obstruction in chronic obstructive pulmonary disease. N Engl J Med 2004;350:2645-2653.

25. Hogg JC, McDonough JE, Suzuki M. Small airway obstruction in COPD: new insights based on micro-CT imaging and MRI imaging. Chest 2013;143:1436-1443.

26. Pozharskaya V, Torres-Gonzalez E, Rojas M, et al. Twist: a regulator of epithelial-mesenchymal transition in lung fibrosis. PLoS One 2009;4:e7559.

27. Bartis D, Mise N, Mahida RY, et al. Epithelial-mesenchymal transition in lung development and disease: does it exist and is it important? Thorax 2013:69:760-765.

28. Resnati M, Guttinger M, Valcamonica $S$, et al. Proteolytic cleavage of the urokinase receptor substitutes for the agonist-induced chemotactic effect. EMBO J 1996;15:1572-1582.

29. Shin VY, Jin $\mathrm{HC}, \mathrm{Ng} E K$, et al. Activation of 5-lipoxygenase is required for nicotine mediated epithelial-mesenchymal transition and tumor cell growth. Cancer Lett 2010;292:237-245.

30. Del RM, Margheri F, Serrati S, et al. The urokinase receptor system, a key regulator at the intersection between inflammation, immunity, and coagulation. Curr Pharm Des 2011;17:1924-1943.

31. Shetty S, Bhandary YP, Shetty SK, et al. Induction of tissue factor by urokinase in lung epithelial cells and in the lungs. Am J Respir Crit Care Med 2010;181:1355-1366.

32. Kuramoto E, Nishiuma T, Kobayashi K, et al. Inhalation of urokinase-type plasminogen activator reduces airway remodeling in a murine asthma model. Am J Physiol Lung Cell Mol Physiol 2009;296:L337-L346.

33. Schmitt M, Harbeck N, Brunner N, et al. Cancer therapy trials employing level-of-evidence-1 disease forecast cancer biomarkers UPA and its inhibitor PAI-1. Expert Rev Mol Diagn 2011;11:617-634.

34. Liang $O D$, Chavakis $T$, Linder $M$, et al. Binding of urokinase plasminogen activator to gp130 via a putative urokinase-binding consensus sequence. Biol Chem 2003;384:229-236.

35. Franco P, Vocca I, Carriero MV, et al. Activation of urokinase receptor by a novel interaction between the connecting peptide region of urokinase and alpha v beta 5 integrin. J Cell Sci 2006;119:3424-3434.

36. Manetti M, Rosa I, Milia AF, et al. Inactivation of urokinase-type plasminogen activator receptor (UPAR) gene induces dermal and pulmonary fibrosis and peripheral microvasculopathy in mice: a new model of experimental scleroderma. Ann Rheum Dis 2014;73: 1700-1709. 